\title{
Real-world data for golimumab treatment in patients with ulcerative colitis in Japan: interim analysis in post-marketing surveillance
}

\author{
Shiro Nakamura ${ }^{1}$, Teita Asano ${ }^{2}$, Hiroaki Tsuchiya ${ }^{2}$, Kanami Sugimoto ${ }^{2}$, Yuya Imai ${ }^{2}$, Seiji Yokoyama ${ }^{2}$, Yasuo Suzuki ${ }^{38}$ \\ ${ }^{1}$ Department of Internal Medicine II, Osaka Medical College Hospital, Takatsuki; ${ }^{2}$ Janssen Pharmaceutical K.K., Tokyo; ${ }^{3}$ Inflammatory Bowel \\ Disease Center, Toho University Sakura Medical Center, Sakura, Japan
}

Background/Aims: Golimumab (GLM) is an anti-tumor necrosis factor- $\alpha$ drug approved for treating moderate-to-severe active ulcerative colitis (UC). A 52-week post-marketing surveillance (PMS) was initiated to evaluate its safety and effectiveness in patients with UC in Japan. We present an interim report of the ongoing PMS. Methods: Patients received $200 \mathrm{mg}$ of subcutaneous GLM at week $0,100 \mathrm{mg}$ at week 2, and $100 \mathrm{mg} 4$ weekly thereafter. The safety analysis set included 392 patients with UC, and the effectiveness analysis set 387 patients. Safety and effectiveness were assessed at week 6 . Results: Adverse drug reactions (ADRs) were reported in 8.2\% (32/392) and serious ADRs in 4.6\% (18/392). The most frequent ADRs were infection and infestation (3.3\%), with herpes zoster being the most common. ADRs were significantly higher in patients with concomitant corticosteroid use (odds ratio [OR], 3.45; 95\% confidence interval [CI], 1.40-9.68). No significant difference in ADR incidence was observed between patients aged $\geq 65$ and $<65$ years (OR, 1.23; 95\% CI, 0.35-3.47). Six-week effectiveness of GLM was confirmed by a decrease in the partial Mayo score $(-2.3 ; 95 \%$ CI, -2.6 to -2.1$)$ and C-reactive protein levels $(-0.64 ; 95 \%$ CI, -0.92 to -0.36), including in the biologics-experienced population. Conclusions: The safety and effectiveness of GLM at week 6 in a real-world setting were demonstrated in patients with UC in Japan. ADR patterns were consistent with previous reports with no new safety signals. Concomitant corticosteroid use may be associated with increased ADR incidence. The final results of the ongoing PMS are necessary for further evaluation. (Intest Res 2022;20:329-341)

Key Words: Golimumab; Colitis, ulcerative; Product surveillance, postmarketing; Safety and effectiveness

\section{INTRODUCTION}

Ulcerative colitis (UC) is a chronic inflammatory disease featuring a pattern of remission and recurrence of mucosal inflammation, starting in the rectum and extending to the proximal colon. The characteristic symptoms and clinical signs in-

Received February 23, 2021. Revised May 22, 2021. Accepted June 7, 2021. Correspondence to Yasuo Suzuki, Inflammatory Bowel Disease Center, Toho University Sakura Medical Center, 564-1, Shimoshizu, Sakura 285-8741, Japan. Tel: +81-3-4411-5031, E-mail: yasuo-suzuki@sakura.med.toho-u.ac.jp Co-Correspondence to Teita Asano, Janssen Pharmaceutical K.K., 5-2, Nishikanda 3-chome, Chiyoda-ku, Tokyo 101-0065, Japan. Tel: +81-3-44117700,E-mail: tasano8@its.jnj.com

${ }^{5}$ Current affiliation: Ginza Central Clinic, Tokyo, Japan. clude diarrhea, abdominal pain, fecal urgency, and rectal bleeding caused by damage to the mucosa. ${ }^{2} \mathrm{UC}$ affects mainly working-age patients and can negatively affect their work productivity and quality of life. ${ }^{3,4}$

Although the exact etiology of UC is not fully understood, the pathogenesis is known to be multifactorial, involving genetic predisposition, environmental factors, epithelial barrier defects, and dysregulated immune responses. ${ }^{1,2}$ Suppressing the dysregulated and excess immune responses to induce and then maintain symptomatic remission and endoscopic healing is important for the treatment of UC. ${ }^{5}$ Immunotherapies currently available, depending on disease extent and severity, include 5-aminosalicylic acid drugs (5-ASAs), corticosteroids, 
thiopurines, calcineurin inhibitors, a Janus kinase inhibitor, and biologics such as anti-tumor necrosis factor (TNF)- $\alpha$ drugs, anti-integrins drugs, and the new anti-interleukin (IL)12/IL-23p40 drug. ${ }^{6}$ Anti-TNF- $\alpha$ drugs, in particular, are widely used for patients with moderate to severely active UC, including those with corticosteroid-dependency and -resistance or inadequate response to thiopurines. The mechanism of antiTNF- $\alpha$ drugs involves blocking soluble and membrane TNF- $\alpha$, which leads to neutralization of a TNF-induced inflammatory response and apoptosis of inflammatory cells. ${ }^{10,11}$ Three antiTNF- $\alpha$ drugs are approved for UC treatment (infliximab [IFX], adalimumab [ADA], and golimumab [GLM]).

GLM is a fully human IgG1 kappa monoclonal anti-TNF- $\alpha$ antibody, ${ }^{12}$ used worldwide to treat rheumatoid arthritis, ${ }^{13}$ psoriatic arthritis, ${ }^{14}$ ankylosing spondylitis, ${ }^{15}$ and juvenile idiopathic arthritis. ${ }^{16}$ GLM is also the third anti-TNF- $\alpha$ drug approved for treating moderate-to-severe active UC, based on the results of the PURSUIT programs. ${ }^{17-19}$ In Japan, GLM is administered subcutaneously at a dose of $200 \mathrm{mg}$ at week $0,100 \mathrm{mg}$ at week 2 for induction, and $100 \mathrm{mg}$ every 4 weeks as UC maintenance therapy. ${ }^{19,20}$ Its subcutaneous route and monthly administration seem to be an advantage in terms of patient compliance. ${ }^{21}$ Clinical trials have demonstrated the safety and efficacy of GLM to induce and maintain a clinical response or remission and induce mucosal healing in patients naïve to anti-TNF- $\alpha$ drugs. ${ }^{17-19}$ Moreover, several recent studies have provided realworld evidence for GLM. ${ }^{22,23}$ An open-label, single-arm phase 4 study in the United Kingdom, GO-COLITIS, reported an effectiveness and safety of GLM in anti-TNF- $\alpha$ naïve patients similar to those observed in the clinical trials. ${ }^{22}$ Although the safety and effectiveness of GLM in real-world settings have been shown by these reports, the available evidence in a large number of patients is still limited, especially in the anti-TNF- $\alpha$ experienced population. In addition, the factors affecting the safety and effectiveness of GLM have not been fully examined.

We conducted a prospective observational study to evaluate real-world safety and effectiveness of GLM in patients with UC through an analysis of interim data from post-marketing surveillance (PMS) in Japan. We also performed a post hoc analysis to examine potential factors that may affect the safety and effectiveness of GLM.

\section{METHODS}

\section{Study Design, Setting, and Patients}

This is a prospective observational study to evaluate the safety and effectiveness of GLM in patients with UC through an analysis of PMS data in Japan. The study consists of 52 weeks with an additional 3-year observational period after initiating treatment in GLM. The study was initiated in May 2017 and will be completed in December 2022. This is the interim report of a series of analyses of the ongoing study.

The patients with UC in about 250 medical facilities based on the plan across Japan were enrolled in the PMS during a 28-month enrollment period. The target number for analysis was 300 patients treated with at least 1 dose of GLM. This sample size was calculated to provide a power of $95 \%$ or more to detect 1 patient reporting an adverse event (AE; defined as any unfavorable and unintended sign, symptom, or disease temporally associated with the use of a drug, and does not imply any judgment about causality) at an incidence of $1.0 \%$. Patients with UC from participating sites who received GLM were centrally registered. Safety and effectiveness data were collected in case report forms after the start of GLM treatment. GLM was administered based on the approved dosage under the real-world clinical practice. In Japan, the approved dosage of subcutaneous GLM is $200 \mathrm{mg}$ at week $0,100 \mathrm{mg}$ at week 2 and 6 , followed by $100 \mathrm{mg}$ every 4 weeks.

The protocol was approved by the Japanese Pharmaceuticals and Medical Devices Agency (PMDA; No. SMP4L) before study initiation. All study procedures were adhered to Good Post-marketing Study Practice (GPSP) in Japan. Because the study complied with the GPSP guidance, institutional review board approval and written informed patient consent were not required. This is currently registered on the University Hospital Medical Information Network Clinical Trials Registry (UMIN-CTR, Identifier: UMIN000027542).

\section{Assessment of the Safety of GLM}

Investigators recorded $\mathrm{AE}$, serious $\mathrm{AEs}$, adverse drug reactions (ADRs; defined as AEs for which a causal relationship to the treatment with the drug cannot be ruled out) and serious ADRs (SADRs) for safety assessments. Safety data were coded using Medical Dictionary for Regulatory Activities version 22.1 and classified by System Organ Class (SOC) and Preferred Term (PT). In addition to patient characteristics, disease status, previous therapy, and concomitant drugs, information related to AEs and ADRs including symptoms, abnormal findings, date of onset, seriousness, dose reduction or discontinuation of GLM, treatment of AEs, causal relationship with GLM, outcomes, and relevant clinical laboratory findings were collected. In particular, the following events were determined as the 
safety specifications based on the Risk Management Plan for GLM: the occurrence of infections and infestations, including pneumonia, tuberculosis, nontuberculosis mycobacterial infection and pneumocystis pneumonia (PCP), as well as malignancy, hematologic disorders, immune system disorders including autoimmune diseases and serious allergic reactions, cardiac disorders, interstitial lung disease, hepatobiliary disorders, and demyelinating diseases.

\section{Assessment of the Effectiveness of GLM}

Disease activity was evaluated for effectiveness assessments using the partial Mayo (pMayo) score, ${ }^{24}$ overall assessment, biochemical markers (fecal calprotectin and C-reactive protein [CRP]), and endoscopic findings. The change in the pMayo score from baseline to week 6 was assessed. Patients, except for those with a pMayo score 0 or 1 at baseline, were further classified into 3 subgroups based on the pMayo score at week 6 (group 1, score 0 or 1; group 2, a decrease from baseline to week 6 greater than 2; and group 3, no decrease or increase from baseline to week 6 ). The proportion of patients in each group was also investigated.

The possible effect of disease activity on the efficacy of GLM was examined by stratifying patients into 3 groups (mild, moderate, and severe) according to Japanese diagnosis criteria, ${ }^{25}$ which is a slightly modified Truelove-Witts index ${ }^{26}$ and the proportion of patients in each group was investigated. Briefly, disease severity was classified based on the following 6 criteria: (1) $\geq 6$ bowel movement/day, (2) bloody stools, (3) pyrexia of $\geq 37.5^{\circ} \mathrm{C}$, (4) pulse rate $\geq 90$ beats $/ \mathrm{min}$, (5) hemoglobin $\leq 10 \mathrm{~g} / \mathrm{dL}$, and/or (6) erythrocyte sedimentation rate $\geq 30 \mathrm{~mm} / \mathrm{hr}$ or CRP $\geq 3.0 \mathrm{mg} / \mathrm{dL}$. Patients were classified as severe if they presented both (1) and (2) plus at least 1 of (3) or (4), and met 4 or more out of the 6 criteria described above. Patients with $\leq 4$ bowel movement/day and/or blood in stools were classified as mild. Patients who showed disease activity between mild and severe were classified as moderate. An overall assessment was performed as "response," "partial response," "no response" or "not evaluable" by the physician in charge. The allowance of the effectiveness data obtained within 4 weeks before or after week 6 were set as the representative values at week 6 .

\section{Statistical Analysis}

The safety analysis set included data from patients who had received at least 1 dose of GLM except patient with protocol violation, and the effectiveness analysis set excluded patients with incomplete data.

Change from baseline in the pMayo score and CRP were tested with the paired $t$-test. Forest plot analyses were used to determine the patient factors affecting the change in the pMayo score. Patients were categorized by age into subgroups according to the Japanese guidelines: elderly ( $\geq 65$ years) and non-elderly ( $<65$ years). ${ }^{25,27}$ Patient factors analyzed for the pMayo score included elderly ( $\geq 65$ years), prior use of biologics, and concomitant medications. In the analysis, 2-group comparisons of mean values were tested with the independent $t$-test. Finally, the independent associated factors were identified by multivariable analysis.

In terms of safety, forest plot analyses for the frequency of ADRs were conducted using the safety analysis set. Two categories of factors were assessed by Fisher exact test. Logistic regression models were also used to determine the independent associated factors.

All statistical analyses were performed using SAS software, version 9.4 (SAS Institute Japan Ltd., Tokyo, Japan), without any imputation for missing data, per the user's manual (SAS Institute I. SAS/STAT user's guide. SAS. 2017; 9.4). The significance level in various tests was set at $5 \%$ (two-sided).

\section{RESULTS}

\section{Patient Demographics and Baseline Characteristics}

A total of 393 patients with UC in Japan were registered during the enrollment period to investigate the safety and effectiveness of GLM in the real-world setting. The case report forms from these 393 patients at week 6 were collected for the study's interim analysis (Fig. 1). All the patients, except for 1 patient due to protocol violation, were included in the safety analysis dataset. Five patients were excluded from the effectiveness analysis data set due to incomplete data, and a final 387 patients were included to assess effectiveness ( $\mathrm{n}=387)$ : pMayo score $(n=376)$, and serum CRP levels $(n=348)$.

A summary of the demographics and baseline characteristics of all 392 patients included in this study is presented in Table 1. The mean \pm standard deviation (SD) age was $43.2 \pm$ 17.1 years, and $13.3 \%$ of the patients were 65 years or older. The mean \pm SD duration of disease was $7.7 \pm 7.6$ years, and $74.0 \%$ had a diagnosis of pancolitis. Intractable UC was present in $88.0 \%$ of patients, of which $66.7 \%$ were steroid-dependent, and $28.4 \%$ were steroid-resistant. The mean \pm SD pMayo score at baseline was $4.9 \pm 2.1$.

Most patients included in this study had previously used 


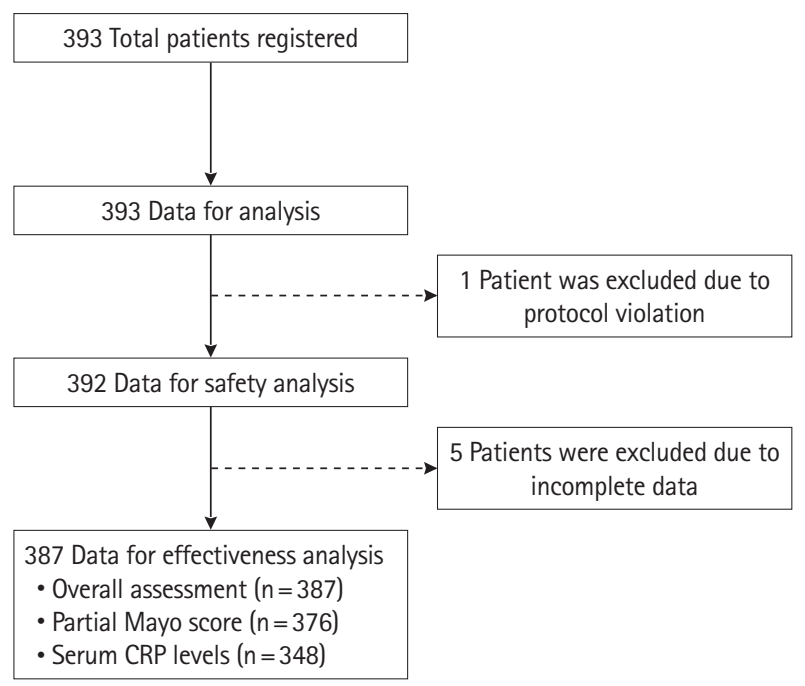

Fig. 1. Disposition of the patients analyzed in this study. In our study, 393 patients were registered for starting golimumab (GLM) therapy. These patients whose case report forms (CRFs) had been collected at week 6 were included in the data set. One patient was excluded due to protocol violation. Therefore, 392 CRFs from patients with ulcerative colitis were analyzed for safety of GLM. The analysis of the effectiveness of GLM was performed after exclusion of 5 patients because of incomplete data. Overall assessment, pMayo score, and serum C-reactive protein (CRP) levels were evaluated in the indicated number of patients.

various medications for UC treatment, and almost all were taking 5-ASA (98.0\%) and corticosteroids (94.1\%). Approximately $70 \%$ of patients had used immunomodulators (IMs), such as azathioprine (AZA) and 6-mercaptopurine (6-MP), and some patients had taken tacrolimus (19.1\%). In addition, $56.9 \%$ had used biologics; $43.1 \%$ had used 1 biologic, and $13.8 \%$ 2 or more. Regarding biologics (anti-TNF- $\alpha$ drugs), $69.1 \%$ had previously used IFX and 50.2\% ADA. The reasons for discontinuation of the biologics included loss of response and AEs; $61.7 \%$ (95/154 patients) and 27.3\% (42/154 patients) for IFX, and $83.0 \%$ (93/112 patients) and 11.6\% (13/112 patients) for ADA, respectively. As for concomitant therapies, the proportion of those concomitantly using 5-ASA, corticosteroids, and IMs (AZA and 6-MP) was $84.4 \%, 55.2 \%$, and $40.1 \%$, respectively. Tacrolimus was used concomitantly in $4.2 \%$. Concomitant use of steroids was recorded in $3.4 \%$ for intravenous corticosteroids, $75.5 \%$ oral, and $33.2 \%$ topical corticosteroids.

\section{Safety}

The incidence of ADRs in the induction periods of GLM is shown in Table 2. In total, 32 patients (8.2\%) developed ADRs, including 18 patients (4.6\%) with SADRs in the safety analysis
Table 1. Patient Demographics and Baseline Characteristics

\begin{tabular}{|c|c|}
\hline Variable & Value $(n=392)$ \\
\hline \multicolumn{2}{|l|}{ Patients characteristics } \\
\hline \multicolumn{2}{|l|}{ Sex } \\
\hline Male & $200(51.0)$ \\
\hline Female & $192(49.0)$ \\
\hline Age (yr) & $43.2 \pm 17.1$ \\
\hline$\geq 65 \mathrm{yr}$ & $52(13.3)$ \\
\hline Body weight (kg) & $57.1 \pm 12.1$ \\
\hline Duration of UC (yr) & $7.7 \pm 7.6$ \\
\hline $\mathrm{BMI}\left(\mathrm{kg} / \mathrm{m}^{2}\right)$ & $21.2 \pm 3.7$ \\
\hline \multicolumn{2}{|l|}{ Smoking history } \\
\hline No & $278(70.9)$ \\
\hline Yes & $74(18.9)$ \\
\hline Continue to smoke & $19(4.9)$ \\
\hline \multicolumn{2}{|l|}{ Disease type } \\
\hline \multicolumn{2}{|l|}{ Disease extent } \\
\hline Pancolitis & $290(74.0)$ \\
\hline Left-sided colitis & $83(21.2)$ \\
\hline Proctitis & $15(3.8)$ \\
\hline Right-sided or segmental colitis & $2(0.5)$ \\
\hline Others & $3(0.8)$ \\
\hline Intractable UC & $345(88.0)$ \\
\hline Steroid resistance & $98(28.4)$ \\
\hline Steroid dependence & $230(66.7)$ \\
\hline Others & $17(4.9)$ \\
\hline \multicolumn{2}{|l|}{ Severity $^{\mathrm{a}}$} \\
\hline Mild & $37(9.4)$ \\
\hline Moderate & 325 (82.9) \\
\hline Severe & $30(7.7)$ \\
\hline \multicolumn{2}{|l|}{ Previous use of non-biologic treatment } \\
\hline $5-A S A$ & $384(98.0)$ \\
\hline Corticosteroids & $369(94.1)$ \\
\hline $\mathrm{AZA}$ & $239(61.0)$ \\
\hline 6-MP & $44(11.2)$ \\
\hline Tacrolimus & $75(19.1)$ \\
\hline \multicolumn{2}{|l|}{ Previous use of biologics } \\
\hline \multicolumn{2}{|l|}{ No. of biologics } \\
\hline 0 & $169(43.1)$ \\
\hline 1 & $169(43.1)$ \\
\hline$\geq 2$ & $54(13.8)$ \\
\hline Biologics type & $223(56.9)$ \\
\hline IFX & $154(69.1)$ \\
\hline IFX-BS & $9(4.0)$ \\
\hline $\mathrm{ADA}$ & $112(50.2)$ \\
\hline
\end{tabular}


Table 1. Continued

\begin{tabular}{lc}
\hline Variable & Value $(\mathrm{n}=\mathbf{3 9 2})$ \\
\hline Concomitant medication during the study & \\
5-ASA & $318(84.4)$ \\
Corticosteroids & $208(55.2)$ \\
Intravenous & $7(3.4)$ \\
Oral & $157(75.5)$ \\
Topical & $69(33.2)$ \\
AZA & $130(34.5)$ \\
6-MP & $21(5.6)$ \\
Tacrolimus & $16(4.2)$ \\
Antibiotics & $13(3.5)$ \\
\hline
\end{tabular}

Values are presented as number (\%) or mean \pm standard deviation.

${ }^{a}$ Baseline severity (mild, moderate, and severe) according to the Japanese diagnosis criteria. ${ }^{25}$

UC, ulcerative colitis; BMI, body mass index; 5-ASA, 5-aminosalicylic acid drugs; AZA, azathioprine; 6-MP, 6-mercaptopurine; IFX, infliximab; IFX$B S$, infliximab biosimilar; $A D A$, adalimumab.

set $(\mathrm{n}=392)$. The most common ADR by SOC was infection and infestations (3.3\%), with herpes zoster being the most frequently observed infection (1.3\%). Skin and subcutaneous tissue disorders by SOC developed in $1.5 \%$ of patients, including alopecia $(0.3 \%)$ and rash $(0.8 \%)$ by PT. Other ADRs observed in $\geq 0.5 \%$ of patients were UC $(0.8 \%)$, rash $(0.8 \%)$, pneumonia $(0.5 \%)$, and pain in an extremity $(0.5 \%)$. UC was the most common SADR and was observed in 3 patients $(0.8 \%)$. Hepatic abnormal function, platelet count decreased, shock, melena, and interstitial lung disease were observed as a single SADR in 1 patient. Tuberculosis, cardiac disorders, injection site reaction, and demyelinating diseases were not observed during the 6-week period of this interim analysis.

We conducted subgroup analysis for the frequency of ADRs (Supplementary Fig. 1). According to the Japanese guideline, we categorized patients into 2 subgroups of elderly ( $\geq 65$ years) and non-elderly ( $<65$ years). ${ }^{25,27}$ No significant difference was observed in the incidence rate of ADRs between elderly and non-elderly patients. The incidence of ADRs was significantly higher in those who concomitantly used corticosteroids compared with those who did not (odds ratio [OR], 3.45; 95\% confidence interval [CI], 1.40-9.68). Combining IMs with GLM did not affect the frequency of ADRs, and neither did previous biologics use. The multivariable analysis also showed that concomitant use of corticosteroids was associated with an increased risk of incidence of ADRs (OR, 3.43; 95\% CI, 1.40-9.68) (Fig. 2). Patients who experienced ADRs were also stratified
Table 2. Incidence of ADRs during the Observation Period $(n=392)$

\begin{tabular}{|c|c|c|}
\hline $\begin{array}{l}\text { ADRs by } \\
\text { System Organ Class classification }\end{array}$ & $\begin{array}{l}\text { SADRs, } \\
\text { No. (\%) }\end{array}$ & $\begin{array}{l}\text { All ADRs, } \\
\text { No. }(\%)\end{array}$ \\
\hline Total number of patients & $18(4.6)$ & $32(8.2)$ \\
\hline Total number of events & 19 & 37 \\
\hline Infections and infestations & $9(2.3)$ & $13(3.3)$ \\
\hline Herpes zoster & $2(0.5)$ & $5(1.3)$ \\
\hline Nasopharyngitis & - & $1(0.3)$ \\
\hline Pneumonia & $2(0.5)$ & $2(0.5)$ \\
\hline Pneumonia legionella & $1(0.3)$ & $1(0.3)$ \\
\hline Anal abscess & $1(0.3)$ & $1(0.3)$ \\
\hline Enteritis infectious & $1(0.3)$ & $1(0.3)$ \\
\hline Pneumonia bacterial & $1(0.3)$ & $1(0.3)$ \\
\hline Listeria sepsis & $1(0.3)$ & $1(0.3)$ \\
\hline Eye disorders & - & $1(0.3)$ \\
\hline Ocular hyperaemia & - & $1(0.3)$ \\
\hline Vascular disorders & $1(0.3)$ & $2(0.5)$ \\
\hline Jugular vein thrombosis & - & $1(0.3)$ \\
\hline Shock & $1(0.3)$ & $1(0.3)$ \\
\hline Respiratory, thoracic and mediastinal disorders & $1(0.3)$ & $1(0.3)$ \\
\hline Interstitial lung disease & $1(0.3)$ & $1(0.3)$ \\
\hline Gastrointestinal disorders & $4(1.0)$ & $4(1.0)$ \\
\hline Colitis ulcerative & $3(0.8)$ & $3(0.8)$ \\
\hline Diarrhea & - & $1(0.3)$ \\
\hline Melaena & $1(0.3)$ & $1(0.3)$ \\
\hline Hepatobiliary disorders & $1(0.3)$ & $1(0.3)$ \\
\hline Hepatic function abnormal & $1(0.3)$ & $1(0.3)$ \\
\hline Skin and subcutaneous tissue disorders & $2(0.5)$ & $6(1.5)$ \\
\hline Acne & - & $1(0.3)$ \\
\hline Alopecia & - & $1(0.3)$ \\
\hline Drug eruption & - & $1(0.3)$ \\
\hline Rash & $2(0.5)$ & $3(0.8)$ \\
\hline Musculoskeletal and connective tissue disorders & - & $2(0.5)$ \\
\hline Pain in extremity & - & $2(0.5)$ \\
\hline $\begin{array}{l}\text { General disorders and administration site } \\
\text { conditions }\end{array}$ & - & $2(0.5)$ \\
\hline Gait disturbance & - & $1(0.3)$ \\
\hline Malaise & - & $1(0.3)$ \\
\hline Investigations & $1(0.3)$ & $3(0.8)$ \\
\hline Blood creatinine increased & - & $1(0.3)$ \\
\hline Blood lactate dehydrogenase increased & - & $1(0.3)$ \\
\hline C-reactive protein increased & - & $1(0.3)$ \\
\hline Platelet count decreased & $1(0.3)$ & $1(0.3)$ \\
\hline
\end{tabular}

Safety data were coded using Medical Dictionary for Regulatory Activities version 22.1 and classified by System Organ Class and Preferred Term. $A D R$, adverse drug reaction; SADR, serious ADR. 


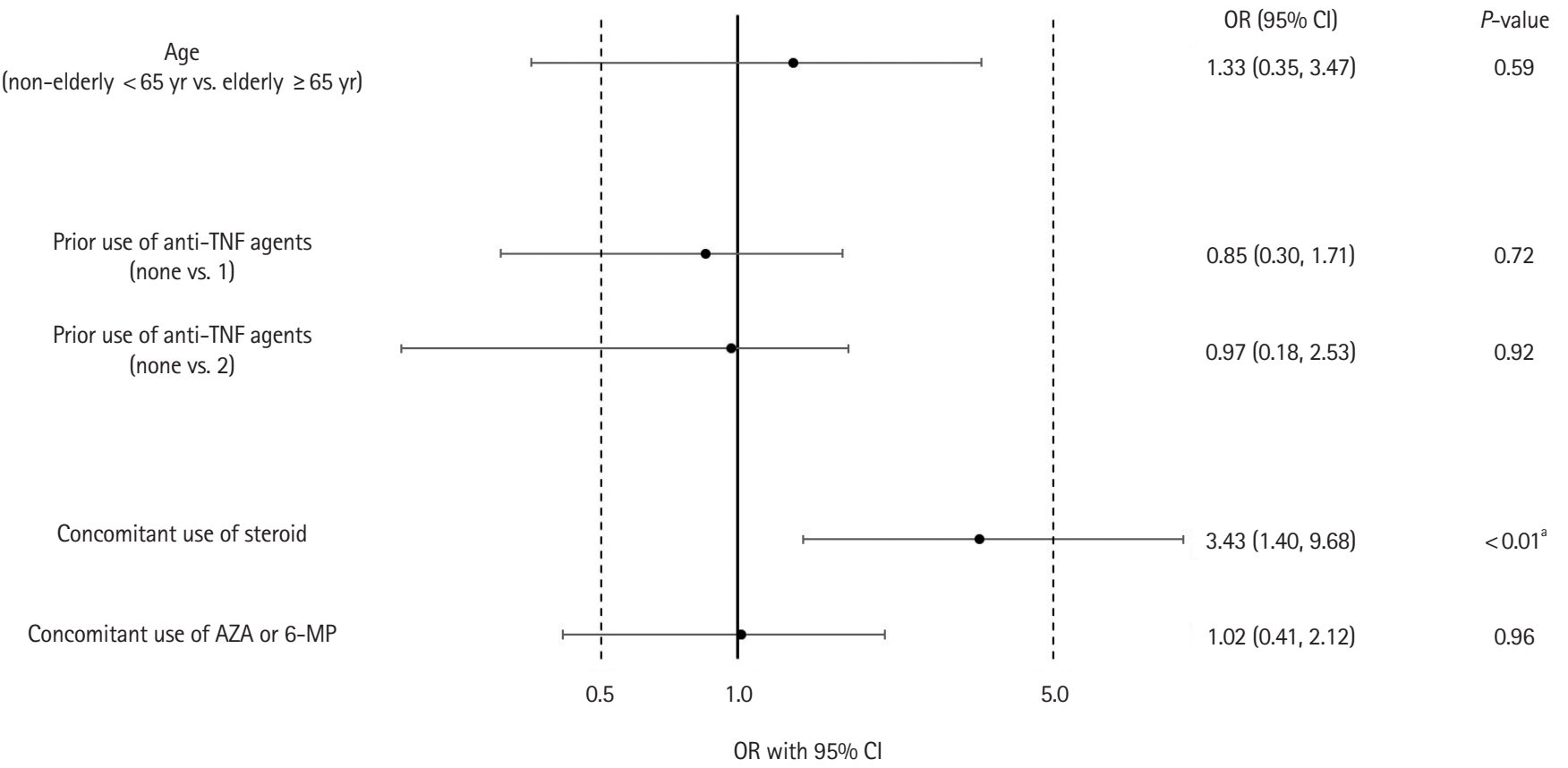

Fig. 2. Multivariable analysis of factors associated with the frequency of adverse drug reactions (ADRs). The patient factors affecting the incidence of ADRs were analyzed using a multivariable logistic regression model. An odds ratio (OR) greater than 1.0 favors increased incidence of ADR, whereas an OR smaller than 1.0 favors decreased incidence of ADR. Non-elderly or elderly are classified as patients aged $<65$ or $\geq 65$ years, respectively. ${ }^{a} P<0.05$. TNF, tumor necrosis factor; AZA, azathioprine; 6-MP, 6-mercaptopurine; $\mathrm{Cl}$, confidence interval.

by concomitant medications (corticosteroids, AZA/6-MP, or both) (Table 3). Out of all ADRs, $12 \%$ of ADRs were observed in the patients receiving corticosteroids: infection and infestations ( 10 cases) by SOC; herpes zoster (4 cases), nasopharyngitis ( 1 case), pneumonia (3 cases; pneumonia, Legionella pneumonia, and bacterial pneumonia, respectively), anal abscess ( 1 case), and listeria sepsis ( 1 case).

\section{Effectiveness}

Effectiveness after initiation of GLM was assessed based on the change in the pMayo score from baseline to week 6 (Fig. 3A). The mean \pm SD pMayo score decreased from $4.9 \pm 2.1$ to $2.6 \pm$ 2.3 at week 6 . The mean serum CRP level decreased from 1.23 $\mathrm{mg} / \mathrm{dL}$ at baseline to $0.59 \mathrm{mg} / \mathrm{dL}$ at week 6 (Fig. 3B). Patients, excluding those with a pMayo score of 0 or 1 at baseline, were further classified into 3 subgroups based on the pMayo score at week 6 . The percentage of patients in group 1, group 2, and group 3 was $32.4 \%, 62.3 \%$, and $22.9 \%$ (Fig. 3C), respectively. We also examined whether disease activity affects the efficacy of GLM. The severity of UC was classified into mild, moderate, and severe, based on clinical symptoms and signs, and blood tests. ${ }^{25} \mathrm{~A}$ decreases in pMayo scores were observed among patients with each disease activity classification (Supplemen- tary Fig. 2). Thirty-nine patients discontinued GLM therapy by week 6 . The reasons for discontinuation were as follows: inadequate response to GLM (23 cases), AEs (9 cases), transfer to a different hospital (4 cases), patient request (1 case), loss to follow-up (1 case), and other ( 1 case).

Forest plot analyses (Supplementary Fig. 3) showed that neither concomitant use of corticosteroids nor AZA/6-MP significantly affected the effectiveness of GLM in patients with UC. Previous anti-TNF- $\alpha$ use influenced the effectiveness of GLM as determined by a change in the pMayo score $(-1.0$; $95 \% \mathrm{CI},-1.5$ to -0.6$)$. The absence of previous anti-TNF- $\alpha$ drug use favored a decrease in the pMayo score compared with the previous use of 1 or 2 anti-TNF- $\alpha$ drugs. No significant difference was observed in the effectiveness of GLM in patients aged $\geq 65$ years (elderly) and $<65$ years (non-elderly). Moreover, the multivariable analyses confirmed that no prior experience of anti-TNF- $\alpha$ drugs was an independent associated factor affecting a decrease in the pMayo score compared with the previous use of 1 or 2 anti-TNF- $\alpha$ drugs (Fig. 4).

\section{DISCUSSION}

Our interim analysis showed that the effectiveness of GLM 
Table 3. Incidence of ADRs Stratified by Concomitant Medication $(n=392)$

\begin{tabular}{|c|c|c|c|c|c|c|}
\hline \multirow{3}{*}{ ADRs by System Organ Class classification } & \multicolumn{6}{|c|}{ No. of patients (\%) } \\
\hline & \multicolumn{3}{|c|}{ With corticosteroids } & \multicolumn{3}{|c|}{ Without corticosteroids } \\
\hline & Total & AZA (+) & 6-MP (+) & Total & AZA (+) & 6-MP (+) \\
\hline Total number of patients & $25(12.0)$ & $10(14.9)$ & $2(22.2)$ & $7(3.8)$ & - & - \\
\hline Total number of events & 29 & 11 & 2 & 8 & - & - \\
\hline Herpes zoster & $4(1.9)$ & - & - & $1(0.5)$ & - & - \\
\hline Nasopharyngitis & $1(0.5)$ & $1(1.5)$ & - & - & - & - \\
\hline Pneumonia & $1(0.5)$ & - & - & $1(0.5)$ & - & - \\
\hline Pneumonia legionella & $1(0.5)$ & $1(1.5)$ & - & - & - & - \\
\hline Anal abscess & $1(0.5)$ & $1(1.5)$ & - & - & - & - \\
\hline Eye disorders & $1(0.5)$ & - & - & - & - & - \\
\hline Ocular hyperaemia & $1(0.5)$ & - & - & - & - & - \\
\hline Vascular disorders & $2(1.0)$ & $1(1.5)$ & - & - & - & - \\
\hline Jugular vein thrombosis & $1(0.5)$ & - & - & - & - & - \\
\hline Shock & $1(0.5)$ & $1(1.5)$ & - & - & - & - \\
\hline Respiratory, thoracic, and mediastinal disorders & $1(0.5)$ & $1(1.5)$ & - & - & - & - \\
\hline Interstitial lung disease & $1(0.5)$ & $1(1.5)$ & - & - & - & - \\
\hline Gastrointestinal disorders & $3(1.4)$ & $1(1.5)$ & - & $1(0.5)$ & - & - \\
\hline Acne & $1(0.5)$ & - & - & - & - & - \\
\hline Alopecia & $1(0.5)$ & $1(1.5)$ & - & - & - & - \\
\hline Drug eruption & - & - & - & - & - & - \\
\hline Rash & $2(1.0)$ & $1(1.5)$ & - & - & - & - \\
\hline Musculoskeletal and connective tissue disorders & $1(0.5)$ & - & - & - & - & - \\
\hline Pain in extremity & $1(0.5)$ & - & - & - & - & - \\
\hline General disorders and administration site conditions & $2(1.0)$ & - & $1(11.1)$ & - & - & - \\
\hline Gait disturbance & $1(0.5)$ & - & - & - & - & - \\
\hline Malaise & $1(0.5)$ & - & $1(11.1)$ & - & - & - \\
\hline Investigations & $3(1.4)$ & $1(1.5)$ & - & - & - & - \\
\hline Blood creatinine increased & $1(0.5)$ & - & - & - & - & - \\
\hline Blood lactate dehydrogenase increased & $1(0.5)$ & $1(1.5)$ & - & - & - & - \\
\hline C-reactive protein increased & $1(0.5)$ & - & - & - & - & - \\
\hline Platelet count decreased & $1(0.5)$ & - & - & - & - & - \\
\hline
\end{tabular}

Safety data were coded using Medical Dictionary for Regulatory Activities version 22.1 and classified by System Organ Class and Preferred Term. $A D R$, adverse drug reaction; AZA, azathioprine; 6-MP, 6-mercaptopurine. 

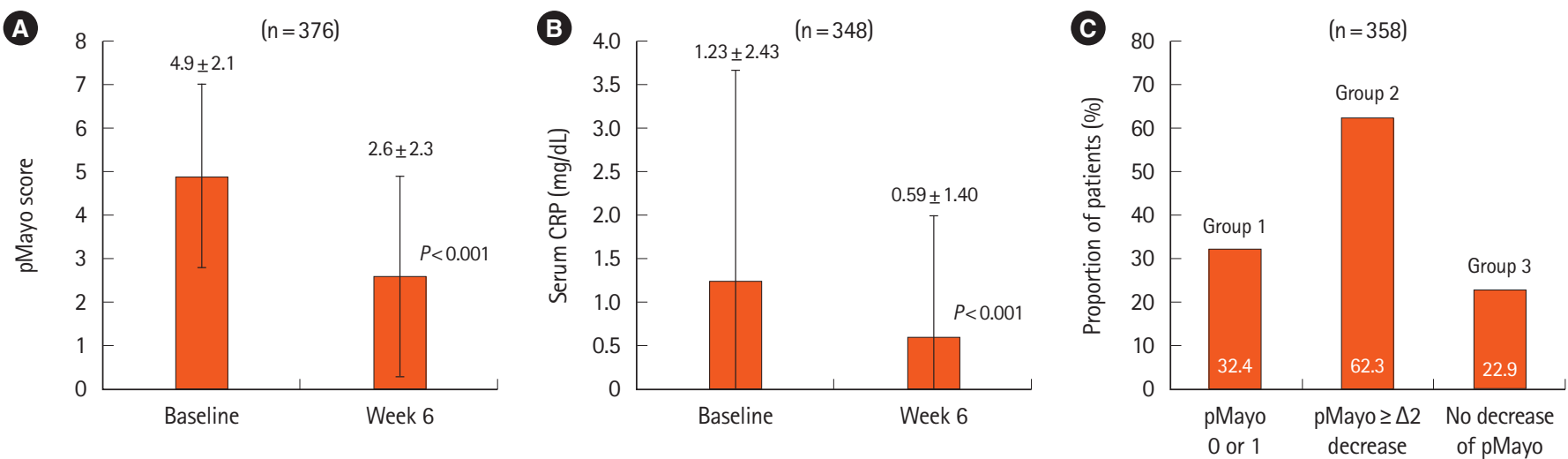

Fig. 3. Effectiveness of golimumab (GLM) after induction treatment. Changes in the pMayo score (A) or CRP (B), and the proportion of the patients in group 1, 2, and 3 (C) were determined by the data from week 0 to 6 . The patients in (A) were classified into 3 subgroups, except for patients who had a pMayo score 0 or 1 at baseline (group 1, score 0 or 1 ; group 2, greater than 2 decrease from baseline to week 6; group 3, no decrease or increase from baseline to week 6). Six-week effectiveness of GLM was confirmed by a decrease from baseline in the partial Mayo score $\left(-2.3 ; 95 \% \mathrm{Cl}_{1}-2.6\right.$ to -2.1$)$ (A) and CRP levels $(-0.64 ; 95 \% \mathrm{Cl},-0.92$ to -0.36$)(\mathrm{B})$ in patients with UC, including the biologics-experienced population. Statistical analysis was performed by paired $t$-test. The values are the mean \pm standard deviation. pMayo, partial Mayo; CRP, C-reactive protein; $\mathrm{Cl}$, confidence interval.

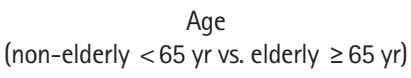

Prior use of anti-TNF agents (none vs. 1)

Prior use of anti-TNF agents (none vs. 2)

Concomitant use of steroid

Concomitant use of AZA or 6-MP

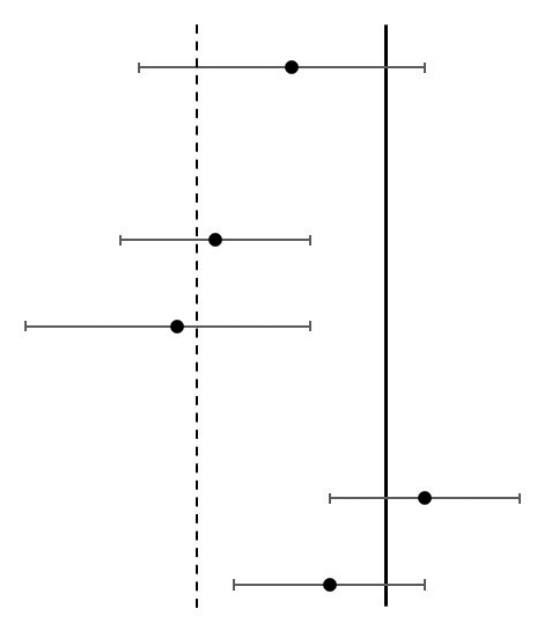

$-1.0$

0.0

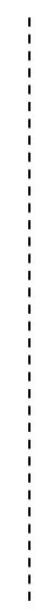

1.0
Means difference $(95 \% \mathrm{Cl})$

$-0.5(-1.3,0.2)$

$-0.9(-1.4,-0.4)$

$-1.1(-1.9,-0.4)$

$0.2(-0.3,0.7)$

$-0.3(-0.8,0.2)$
$P$-value

0.140

$<0.001^{\mathrm{a}}$

$<0.010^{\mathrm{a}}$

0.370

0.210

Mean difference of change from baseline of pMayo score

Fig. 4. Multivariable analysis of factors associated with changes in the pMayo score. The patient factors affecting the effectiveness of golimumab (GLM) treatment were identified by multiple regression analysis. Change in the pMayo score from baseline to week 6 was used to evaluate GLM effectiveness. Non-elderly or elderly classified as patients aged $<65$ or $\geq 65$ years, respectively. ${ }^{a} P<0.05$. TNF, tumor necrosis factor; AZA, azathioprine; 6-MP, 6-mercaptopurine; $\mathrm{Cl}$, confidence interval; pMayo, partial Mayo.

was similar to other real-world experiences, and no new safety concerns were detected.

Similar to other reports, ${ }^{28,29}$ almost all patients in our study were steroid-dependent or steroid-resistant, and more than 70\% had previously taken 5-ASA, corticosteroids, or IMs (AZA/ 6-MP). In line with Japanese guidelines, ${ }^{25}$ our study showed that GLM was used to treat refractory UC patients who failed to or were intolerant to conventional therapy. Furthermore, more than half of the patients had previously received antiTNF- $\alpha$ therapy, who mostly switched to GLM from either IFX or ADA. Corticosteroids were concomitantly used in most patients, and the proportion was higher than those in PURSUIT-J and PURSUIT-SC. Because these clinical trials restricted the dosage of medication use, our results may represent the realworld evidence in Japan. Concomitant use of AZA/6-MP was similar rate between our study and the clinical trials. ${ }^{17,19}$ In our 
study, the pMayo score was lower than other real-world studies. $^{28-30}$

No significant patterns were observed for ADRs, which were numerically comparable to the previous reports. ${ }^{28,29,31}$ Our results showed that the most frequent ADRs and SADRs were infection and UC, respectively. This is in line with the results of PURSUIT-SC and other real-world evidence. ${ }^{17,28,30}$ In addition to this interim analysis, the most recent Japan-Periodic Safety Update Report (cutoff date: April 6, 2020) reported ADRs and serious ADRs occurring in 16.8\% (66/392) and 9.7\% (38/392) of patients, respectively. In this report, the SOC with the most frequently reported AEs was infections and infestations (5.1\%), and the most frequent AEs by PT was herpes zoster infection (1.3\%) (the data are internal documents of Janssen Pharmaceutical). Previous studies showed that immunosuppressive therapies, including anti-TNF- $\alpha$ drug, thiopurines, their combination, and corticosteroids were associated with an increased risk of herpes zoster infection in patients with inflammatory bowel disease. ${ }^{32}$ An increased incidence rate of herpes zoster infection in Asian, but not white and black populations, has been reported in UC patients receiving tofacitinib. ${ }^{33}$ As the present study was performed in a mainly Japanese population, genetic predisposition might be involved in the frequent observation of herpes zoster among infectious diseases.

Our multivariable analysis identified the concomitant use of corticosteroids as an independent associated risk factor for increased ADRs in patients with UC (Fig. 2). As the similar association was observed for AEs with cumulative and average corticosteroids doses, ${ }^{34,35}$ it is likely that cumulative exposure of corticosteroids rather than periods of combined use may be involved in the elevated incidence of ADRs. Similar to our findings, the risk of infection has been reported to be higher when the anti-TNF- $\alpha$ drug is combined with corticosteroids compared to anti-TNF- $\alpha$ drug monotherapy. ${ }^{36}$

IMs have often been used concomitantly for corticosteroid withdrawal; however, this often resulted in failure and the need for additional therapy. Anti-TNF- $\alpha$ therapy combined with steroids and IMs increases opportunistic infection risk compared with combinations with steroids or IMs alone. ${ }^{37}$ Moreover, combining IFX and AZA with corticosteroids has previously been shown to increase the risk of PCP infection and prolonged recovery in patients with inflammatory bowel disease ${ }^{38,39}$ however, this was not observed in our study. In fact, among the 10 infections observed in patients with concomitant corticosteroid use, patients received a combination of both corticosteroids and IMs (AZA/6-MP) (Table 3). How- ever, we could not fully evaluate whether concomitant use of both corticosteroids and IMs may increase infection risk because of the short observational period. Therefore, careful monitoring may still be needed for opportunistic infections, including PCP, when combining GLM with corticosteroids and IMs. Use of sulfamethoxazole-trimethoprim combination for PCP prophylaxis has been suggested for high risk of UC patients. ${ }^{39}$

A previous report suggested that age is 1 of the risk factors for ADRs in UC. ${ }^{40,41}$ Diagnosis and management of elderly patients with UC presents many difficulties compared to those of non-elderly patients, owing to comorbidities (e.g., chronic obstructive pulmonary disease, cardiovascular disease, and diabetes), immunological dysfunction, and polypharmacy (prolonged uses of steroids or immunosuppressive agents), which can contribute to an increased incidence for AEs. ${ }^{27} \mathrm{Cot}-$ tone et al. ${ }^{42}$ reported that IBD patients aged $\geq 65$ years treated with IFX or ADA experienced severe infections more frequently than the same age group without these treatments. In addition, Toruner et al. ${ }^{37}$ reported that immunosuppressive medications, especially steroids and older age, are associated with an increased risk of opportunistic infections. Unlike the previous reports, in this interim analysis, no significant differences were observed between the elderly and non-elderly in terms of the incidence of ADRs during the 6-week observation periods. Although our short-term data suggests no influence of older age on ADR incidence, the ADR risk in elderly patients may increase during long-term observation. The results of our ongoing study might clarify this possibility. Moreover, in this study, we could not clearly distinguish elderly-onset UC patients from elderly patients with UC. Recent studies reported that elderly-onset UC patients showed different clinical features and comorbidities from adult-onset UC. ${ }^{41,43}$

In our study, a significant decrease in the pMayo score and serum CRP levels were observed in patients with UC in Japan, 6 weeks after GLM initiation. The proportion of GLM responders were similar to the pooled analysis of short-term periods (6-14 weeks) reported by Olivera et al. ${ }^{23}$ This study, like other previous reports, also showed that effectiveness was significantly higher in those who were anti-TNF- $\alpha$ drugs naïve than in those who had previously used them. ${ }^{25,38}$ Clinical outcomes have also been reported to be significantly worse in patients receiving GLM who had failed 2 anti-TNF- $\alpha$ treatment. ${ }^{28,44,45}$ This report also showed no significance in GLM effectiveness between anti-TNF- $\alpha$ drug naïve and 1 anti-TNF- $\alpha$ drug experienced patients. ${ }^{44}$ In contrast to this finding, our results showed more effectiveness in the anti-TNF- $\alpha$ naïve group compared to 
1 anti-TNF- $\alpha$ experienced group. In the present study, a separate analysis based on whether drugs were switched due to intolerance or loss of response to prior anti-TNF- $\alpha$ drug was not planned initially because PMS is a part of the additional pharmacovigilance activity required by our regulatory authority and primarily focused on safety assessment. There is a possibility that the different contexts for withdrawal of the prior anti-TNF drugs might be associated with the effectiveness of GLM. Results based on the reason for switching are limited in UC patients. To our knowledge, related results have not been reported in GLM therapy. However, some data have been reported for other anti-TNF therapies. Christensen et al. ${ }^{46}$ found that the patients who switched due to allergic reaction to IFX had a longer persistent and colectomy-free time on ADA treatment than all others. In addition to the findings for UC, the meta-analysis reported by Gisbert et al. ${ }^{47}$ suggested that clinical outcomes were better in patients with intolerance to the first anti-TNF- $\alpha$ drug than those with secondary or primary failure in the patients with $\mathrm{CD}$. These findings suggest better outcomes with GLM treatment in patients with intolerance to previous anti-TNF drugs compared to those with loss of response to the prior anti-TNF drugs. However, it is difficult to discuss due to lack of data. Moreover, we need to consider not only the reason for switching from previous anti-TNF drugs but also other affecting factors, such as previous dose escalation, switching patterns (from IFX to GLM or from ADA to GLM), and the time to switch. ${ }^{48,49}$ Thus, further studies are necessary to evaluate these aspects in detail. Because the PURSUIT trials targeted only anti-TNF- $\alpha$ drug naïve patients, the data for the effectiveness of GLM in UC patients previously exposed to anti-TNF- $\alpha$ drug is limited. To the best of our knowledge, our report is the first real-world evidence for the effectiveness of GLM induction treatment in a larger Asian population, including anti-TNF- $\alpha$ experienced patients. We also determined whether disease activity at baseline influenced GLM effectiveness or not. We confirmed similar effectiveness of GLM in patients grouped by disease activity, as classified by the Japanese diagnosis criteria (Supplementary Fig. 2). As described in the Methods, we evaluated disease severity according to the Japanese guidelines (modified Truelove and Witts criteria). In our study, patients with severe UC met at least 4 out of 6 items in the Truelove and Witts criteria. On the other hand, under the AGA guidelines, patients who have 3 or more out of 6 items in the criteria ( 6 bloody bowel movements/day with at least 1 blood marker or systemic toxicity) are classified as acute severe $U \mathrm{UC}^{50}$ Therefore, the patient group classified as severe in Supplementary Fig. 1 also includes patients with acute severe UC. Because GLM was effective in patients diagnosed with severe UC according to the Japanese guidelines, this suggests that it may also be effective in patients with acute severe UC based on the AGA guidelines. However, further investigation is necessary because of the limited number of patients in our study and the slight difference between these 2 criteria.

Stratification analysis for the effectiveness of GLM demonstrated no significant difference between concomitant steroid use and nonsteroids users at week 6 (Supplementary Fig. 3). In the PURSUIT-SC, concomitantly using corticosteroids was observed to have a higher clinical response rate compared to not using corticosteroids. ${ }^{17}$ This discrepancy might be due to the differences between the designed trials and the real-world setting. As described above, concomitant use of corticosteroids during GLM induction periods may be associated with an increased incidence rate of ADRs, especially infections. Our data suggest that concomitant use of corticosteroids does not confer benefit in patients with UC in clinical practice. Considering these findings, avoiding or tapering corticosteroids when appropriate should be considered with GLM treatment. We also observed no significant influence of IM combination therapy on GLM effectiveness. Further studies are necessary to evaluate these aspects. Older age was also not found to influence GLM efficacy in the stratification analysis. Similarly, other studies have reported that the effectiveness of biologics, including GLM, in elderly patients with UC may be comparable to those in non-elderly, except for elderly-onset UC patients. $^{27,43}$

Our study has several limitations. First, we could not exclude biases due to the absence of a control group and open-label treatment. Second, we could not evaluate endoscopic examination and fecal calprotectin because of lack of data. Mucosal healing is important for evaluating UC treatment. Although the effectiveness of GLM on endoscopic improvement in the patients with UC could not be examined in our study, we confirmed the effectiveness of GLM based on clinical symptoms and the serum inflammatory marker CRP.

Our study's strengths include being a prospective, multicenter, large cohort with patients who had previously used anti-TNF- $\alpha$ drugs providing real-world data in Japan. This contrasts with other studies that are retrospective and single-center in design, include only TNF naïve population, and use a small sample size. The current study is ongoing, so long-term experience data is expected to be available in the future. 
In conclusion, the 6-week safety and effectiveness of GLM in the real-world, not only anti-TNF- $\alpha$ drug naïve but also antiTNF- $\alpha$ drug-exposed patients, were confirmed by our study. Concomitant use of corticosteroids may be associated with an increased risk of ADRs, without providing additional effectiveness. Therefore, corticosteroids should be used cautiously when GLM therapy is initiated, evaluating the potential risks and benefits of effectiveness and complications. However, more long-term results are necessary for further evaluation.

\section{ADDITIONAL INFORMATION}

\section{Funding Source}

Sponsorship for this study were provided by Janssen Pharmaceutical K.K. (Tokyo, Japan) and Mitsubishi Tanabe Pharma Corporation (Osaka, Japan).

\section{Conflict of Interest}

Asano T, Tsuchiya H, Sugimoto K, Imai Y, and Yokoyama S are full-time employees of Janssen Pharmaceutical K.K. Nakamura $S$ has received speaking fees from AbbVie GK, EA Pharma Co., Ltd., Mitsubishi Tanabe Pharma Corporation., Mochida Pharmaceutical Co., Ltd., Takeda Pharmaceutical Co., Ltd, and Janssen Pharmaceutical K.K.; and has served as a consultant for Janssen Pharmaceutical K.K. Suzuki Y has received speaking fees from AbbVie GK, EA Pharma Co., Ltd., Mitsubishi Tanabe Pharma Corporation, Mochida Pharmaceutical Co., Ltd., Kyorin Pharmaceutical Co., Ltd., ZERIA Pharmaceutical Co., Ltd, and Janssen Pharmaceutical K.K.; has served on grants/research support from AbbVie GK, EA Pharma Co., Ltd., Mitsubishi Tanabe Pharma Co., Ltd., Mochida Pharmaceutical Co., Ltd., Kissei Pharmaceutical Co., Ltd., and JIMRO Co., Ltd.; and has served as a consultant for Janssen Pharmaceutical K.K. No other potential conflicts of interest relevant to this article were reported.

\section{Data Availability Statement}

Not applicable.

\section{Author Contribution}

Conceptualization: Tsuchiya H, Imai Y, Yokoyama S. Methodology: Tsuchiya H, Sugimoto K, Imai Y, Yokoyama S. Formal analysis: Nakamura S, Asano T, Tsuchiya H, Imai Y, Yokoyama S, Suzuki Y. Funding acquisition: Tsuchiya H, Imai Y, Suzuki Y. Project administration: Yokoyama S. Visualization: Asano T, Tsuchiya H, Imai Y, Yokoyama S. Writing - original draft: Asano
T, Yokoyama S. Writing - review and editing: Nakamura S, Asano T, Yokoyama S, Tsuchiya H, Sugimoto K, Imai Y. Approval of final manuscript: all authors.

\section{Others}

We deeply appreciate the contribution of the physicians from the participating medical institutions and the patients who participated in this study. We also wish to thank Shinichi Yoshigoe (Immunology and Infectious Diseases \& Vaccine Department, Medical Affairs Division, Janssen Pharmaceutical K.K.) and Noriko Sato (Ikuyaku, Integrated Value Development Division, Mitsubishi Tanabe Pharma Corporation) for their contribution to the study design and data interpretation. We would also like to express our gratitude to Akiko Yamamoto, of Janssen Pharmaceutical K.K., who helped with the execution and management of the study.

All authors had full access to all the data in this study and take complete responsibility for the integrity of the data and accuracy of the data analysis.

\section{ORCID}

Nakamura S $\quad$ https://orcid.org/0000-0003-4435-1294

Asano T https://orcid.org/0000-0002-1830-4382

Tsuchiya H https://orcid.org/0000-0001-6530-7298

Sugimoto K https://orcid.org/0000-0002-1912-9002

Imai Y https://orcid.org/0000-0002-9125-3358

Yokoyama S https://orcid.org/0000-0003-4995-4983

Suzuki Y https://orcid.org/0000-0002-9813-9793

\section{Supplementary Material}

Supplementary materials are available at the Intestinal Research website (https://www.irjournal.org).

\section{REFERENCES}

1. Ungaro R, Mehandru S, Allen PB, Peyrin-Biroulet L, Colombel JF. Ulcerative colitis. Lancet 2017;389:1756-1770.

2. Kobayashi T, Siegmund B, Le Berre C, et al. Ulcerative colitis. Nat Rev Dis Primers 2020;6:74.

3. Ueno F, Nakayama Y, Hagiwara E, Kurimoto S, Hibi T. Impact of inflammatory bowel disease on Japanese patients' quality of life: results of a patient questionnaire survey. J Gastroenterol 2017;52:555-567.

4. Yarlas A, Rubin DT, Panés J, et al. Burden of ulcerative colitis on functioning and well-being: a systematic literature review of the SF-36 health survey. J Crohns Colitis 2018;12:600-609. 
5. Friedrich M, Pohin M, Powrie F. Cytokine networks in the pathophysiology of inflammatory bowel disease. Immunity 2019;50: 992-1006.

6. Harbord M, Eliakim R, Bettenworth D, et al. Third European evidence-based consensus on diagnosis and management of ulcerative colitis. Part 2: current management. J Crohns Colitis 2017;11:769-784.

7. Singh S, Allegretti JR, Siddique SM, Terdiman JP. AGA technical review on the management of moderate to severe ulcerative colitis. Gastroenterology 2020;158:1465-1496.

8. Sands BE, Sandborn WJ, Panaccione R, et al. Ustekinumab as induction and maintenance therapy for ulcerative colitis. $\mathrm{N}$ Engl J Med 2019;381:1201-1214.

9. Sandborn WJ, Su C, Sands BE, et al. Tofacitinib as induction and maintenance therapy for ulcerative colitis. N Engl J Med 2017;376:1723-1736.

10. Mitoma H, Horiuchi T, Tsukamoto H, Ueda N. Molecular mechanisms of action of anti-TNF- $\alpha$ agents: comparison among therapeutic TNF- $\alpha$ antagonists. Cytokine 2018;101:56-63.

11. Billmeier U, Dieterich W, Neurath MF, Atreya R. Molecular mechanism of action of anti-tumor necrosis factor antibodies in inflammatory bowel diseases. World J Gastroenterol 2016; 22:9300-9313.

12. Shealy DJ, Cai A, Staquet K, et al. Characterization of golimumab, a human monoclonal antibody specific for human tumor necrosis factor $\alpha$. MAbs 2010;2:428-439.

13. Kay J, Matteson EL, Dasgupta B, et al. Golimumab in patients with active rheumatoid arthritis despite treatment with methotrexate: a randomized, double-blind, placebo-controlled, dose-ranging study. Arthritis Rheum 2008;58:964-975.

14. Kavanaugh A, McInnes I, Mease P, et al. Golimumab, a new human tumor necrosis factor alpha antibody, administered every four weeks as a subcutaneous injection in psoriatic arthritis: twenty-four-week efficacy and safety results of a randomized, placebo-controlled study. Arthritis Rheum 2009;60: 976-986.

15. Inman RD, Davis JC Jr, Heijde D, et al. Efficacy and safety of golimumab in patients with ankylosing spondylitis: results of a randomized, double-blind, placebo-controlled, phase III trial. Arthritis Rheum 2008;58:3402-3412.

16. Brunner HI, Ruperto N, Tzaribachev N, et al. Subcutaneous golimumab for children with active polyarticular-course juvenile idiopathic arthritis: results of a multicentre, double-blind, randomised-withdrawal trial. Ann Rheum Dis 2018;77:21-29.

17. Sandborn WJ, Feagan BG, Marano C, et al. Subcutaneous golimumab induces clinical response and remission in patients with moderate-to-severe ulcerative colitis. Gastroenterology 2014;146:85-95.

18. Sandborn WJ, Feagan BG, Marano C, et al. Subcutaneous golimumab maintains clinical response in patients with moderateto-severe ulcerative colitis. Gastroenterology 2014;146:96-109.

19. Hibi T, Imai Y, Senoo A, Ohta K, Ukyo Y. Efficacy and safety of golimumab 52-week maintenance therapy in Japanese patients with moderate to severely active ulcerative colitis: a phase 3, double-blind, randomized, placebo-controlled study(PURSUIT-J study). J Gastroenterol 2017;52:1101-1111.

20. Vermeire S, D'heygere F, Nakad A, et al. Preference for a prefilled syringe or an auto-injection device for delivering golimumab in patients with moderate-to-severe ulcerative colitis: a randomized crossover study. Patient Prefer Adherence 2018; 12:1193-1202.

21. Dragoni G, Le Grazie M, Orlandini B, Rogai F. Golimumab in inflammatory bowel diseases: present and future scenarios. Clin J Gastroenterol 2019;12:1-9.

22. Probert CS, Sebastian S, Gaya DR, et al. Golimumab induction and maintenance for moderate to severe ulcerative colitis: results from GO-COLITIS (Golimumab: a phase 4, UK, open label, single arm study on its utilization and impact in ulcerative Colitis). BMJ Open Gastroenterol 2018;5:e000212.

23. Olivera P, Danese S, Pouillon L, Bonovas S, Peyrin-Biroulet L. Effectiveness of golimumab in ulcerative colitis: a review of the real world evidence. Dig Liver Dis 2019;51:327-334.

24. Lewis JD, Chuai S, Nessel L, Lichtenstein GR, Aberra FN, Ellenberg JH. Use of the noninvasive components of the Mayo score to assess clinical response in ulcerative colitis. Inflamm Bowel Dis 2008;14:1660-1666.

25. Matsuoka K, Kobayashi T, Ueno F, et al. Evidence-based clinical practice guidelines for inflammatory bowel disease. J Gastroenterol 2018;53:305-353.

26. Truelove SC, Witts LJ. Cortisone in ulcerative colitis; final report on a therapeutic trial. Br Med J 1955;2:1041-1048.

27. Higashiyama M, Sugita A, Koganei K, et al. Management of elderly ulcerative colitis in Japan. J Gastroenterol 2019;54:571586.

28. Bossa F, Biscaglia G, Valvano MR, et al. Real-life effectiveness and safety of golimumab and its predictors of response in patients with ulcerative colitis. Dig Dis Sci 2020;65:1767-1776.

29. Orlandini B, Dragoni G, Variola A, et al. Clinical efficacy and safety of golimumab in biologically experienced and naïve patients with active ulcerative colitis: a real-life experience from two Italian IBD centers. J Dig Dis 2018;19:468-474.

30. Tursi A, Allegretta L, Buccianti N, et al. Effectiveness and safe- 
ty of golimumab in treating outpatient ulcerative colitis: a reallife prospective, multicentre, observational study in primary inflammatory bowel diseases centers. J Gastrointestin Liver Dis 2017;26:239-244.

31. Simponi Interview Form registered in PMDA search system for prescription drugs [Internet]. c2020 [cited 2020 Sep 25]. https://www.info.pmda.go.jp/downfiles/guide/ph/800155_ 3999433G1024_1_00G.pdf.

32. Long MD, Martin C, Sandler RS, Kappelman MD. Increased risk of herpes zoster among 108604 patients with inflammatory bowel disease. Aliment Pharmacol Ther 2013;37:420-429.

33. Winthrop KL, Melmed GY, Vermeire S, et al. Herpes zoster infection in patients with ulcerative colitis receiving tofacitinib. Inflamm Bowel Dis 2018;24:2258-2265.

34. Curtis JR, Westfall AO, Allison J, et al. Population-based assessment of adverse events associated with long-term glucocorticoid use. Arthritis Rheum 2006;55:420-426.

35. Morin C, Fardet L. Systemic glucocorticoid therapy: risk factors for reported adverse events and beliefs about the drug: a cross-sectional online survey of 820 patients. Clin Rheumatol 2015;34:2119-2126.

36. Singh S, Facciorusso A, Dulai PS, Jairath V, Sandborn WJ. Comparative risk of serious infections with biologic and/or immunosuppressive therapy in patients with inflammatory bowel diseases: a systematic review and meta-analysis. Clin Gastroenterol Hepatol 2020;18:69-81.

37. Toruner M, Loftus EV Jr, Harmsen WS, et al. Risk factors for opportunistic infections in patients with inflammatory bowel disease. Gastroenterology 2008;134:929-936.

38. Seddik M, Meliez H, Seguy D, Viget N, Cortot A, Colombel JF. Pneumocystis jiroveci (carinii) pneumonia following initiation of infliximab and azathioprine therapy in a patient with Crohn's disease. Inflamm Bowel Dis 2004;10:436-437.

39. Kojima K, Sato T, Uchino M, et al. Clinical characteristics and risk factors for pneumocystis jirovecii pneumonia during immunosuppressive treatment in patients with ulcerative colitis: a retrospective study. J Gastrointestin Liver Dis 2020;29:167173.

40. Ananthakrishnan AN, Donaldson T, Lasch K, Yajnik V. Management of inflammatory bowel disease in the elderly patient: challenges and opportunities. Inflamm Bowel Dis 2017;23:
882-893

41. Zammarchi I, Lanzarotto F, Cannatelli R, et al. Elderly-onset vs adult-onset ulcerative colitis: a different natural history? BMC Gastroenterol 2020;20:147.

42. Cottone M, Kohn A, Daperno M, et al. Advanced age is an independent risk factor for severe infections and mortality in patients given anti-tumor necrosis factor therapy for inflammatory bowel disease. Clin Gastroenterol Hepatol 2011;9: 30-35.

43. Komoto S, Higashiyama M, Watanabe C, et al. Clinical differences between elderly-onset ulcerative colitis and non-elderly-onset ulcerative colitis: a nationwide survey data in Japan. J Gastroenterol Hepatol 2018;33:1839-1843.

44. Taxonera C, Rodríguez C, Bertoletti F, et al. Clinical outcomes of golimumab as first, second or third anti-TNF agent in patients with moderate-to-severe ulcerative colitis. Inflamm Bowel Dis 2017;23:1394-1402.

45. Samaan MA, Pavlidis P, Digby-Bell J, et al. Golimumab: early experience and medium-term outcomes from two UK tertiary IBD centres. Frontline Gastroenterol 2018;9:221-231.

46. Christensen KR, Steenholdt C, Brynskov J. Clinical outcome of adalimumab therapy in patients with ulcerative colitis previously treated with infliximab: a Danish single-center cohort study. Scand J Gastroenterol 2015;50:1018-1024.

47. Gisbert JP, Marín AC, McNicholl AG, Chaparro M. Systematic review with meta-analysis: the efficacy of a second anti-TNF in patients with inflammatory bowel disease whose previous anti-TNF treatment has failed. Aliment Pharmacol Ther 2015; 41:613-623

48. Gemayel NC, Rizzello E, Atanasov P, Wirth D, Borsi A. Dose escalation and switching of biologics in ulcerative colitis: a systematic literature review in real-world evidence. Curr Med Res Opin 2019;35:1911-1923.

49. Taxonera C, Iglesias E, Muñoz F, et al. Adalimumab maintenance treatment in ulcerative colitis: outcomes by prior antiTNF use and efficacy of dose escalation. Dig Dis Sci 2017;62: 481-490.

50. Feuerstein JD, Isaacs KL, Schneider Y, et al. Spotlight: management of moderate-to-severe ulcerative colitis. Gastroenterology 2020;158:1464. 
See "Real-world data for golimumab treatment in patients with ulcerative colitis in Japan: interim analysis in post-marketing surveillance" on pages 329-341.

$$
\begin{gathered}
\text { Age } \\
\text { (non-elderly }<65 \text { yr vs. elderly } \geq 65 \text { yr) } \\
\text { Prior use of anti-TNF agents } \\
\text { (no vs. yes) } \\
\text { Prior use of anti-TNF agents } \\
\text { (none vs. 1) } \\
\text { Prior use of anti-TNF agents } \\
\text { (none vs. 2) } \\
\text { Prior use of anti-TNF agents } \\
\text { (1 vs. 2) } \\
\text { Concomitant use of steroid } \\
\text { Concomitant use of AZA or 6-MP }
\end{gathered}
$$

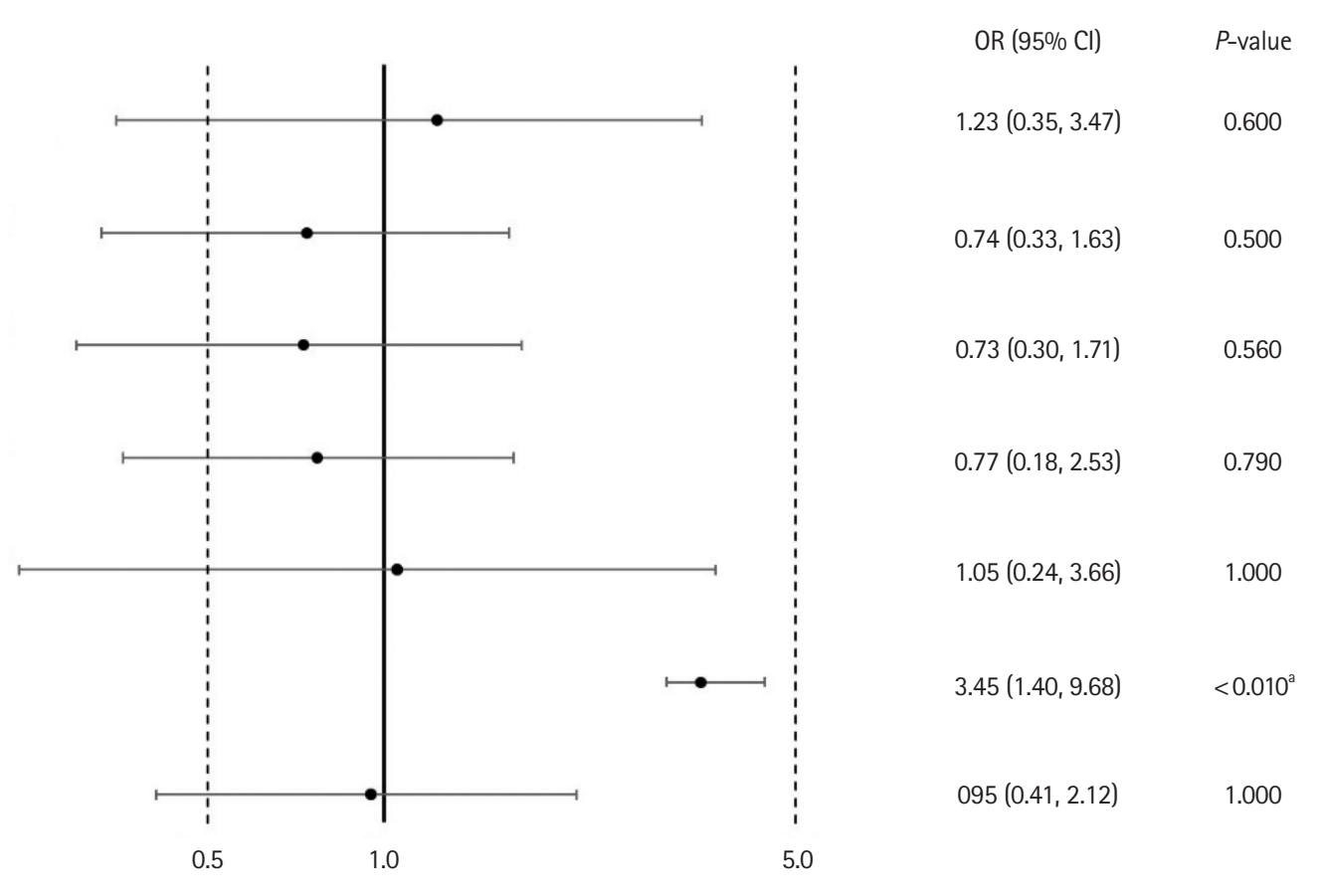

OR wiht $95 \% \mathrm{Cl}$

Supplementary Fig. 1. Univariable analysis of the factors associated with the frequency of adverse drug reactions (ADRs). The patient factors affecting the incidence of ADRs were demonstrated with forest plots. An odds ratio (OR) greater than 1.0 favors increased incidence of ADR, whereas an OR smaller than 1.0 favors decreased incidence of ADR. Non-elderly or elderly classified as patients aged $<65$ or $\geq 65$ years, respectively. The $P$-value was calculated using the Fisher exact test. ${ }^{a} P<0.05$. TNF, tumor necrosis factor; AZA, azathioprine; 6-MP, 6-mercaptopurine; $\mathrm{Cl}$, confidence interval. 
A

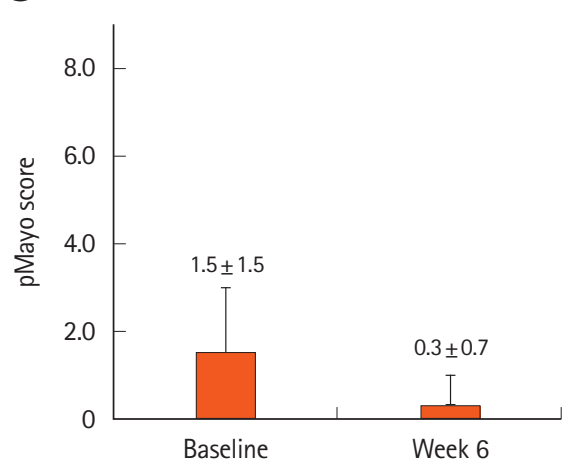

B

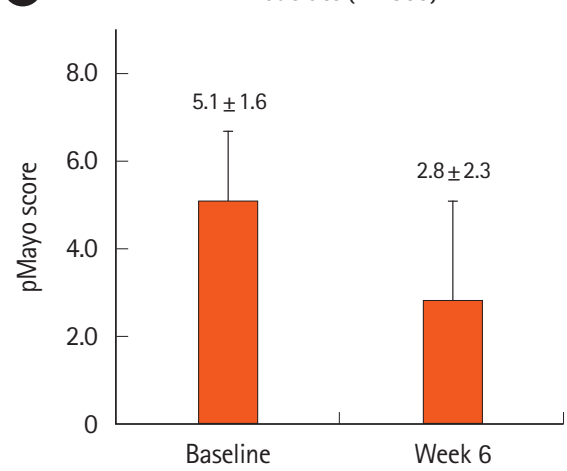

C

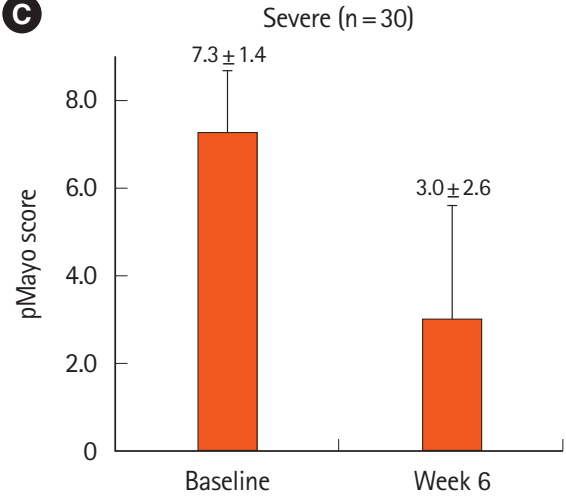

Supplementary Fig. 2. The mean partial Mayo (pMayo) score in patients with different disease activity at baseline and 6 weeks after golimumab treatment $(n=376)$. Patients with pMayo score were stratified into 3 groups (mild, moderate, and severe) classified by the disease severity at baseline according to the Japanese diagnosis criteria. ${ }^{1}$ The mean pMayo score ( \pm standard deviation) at baseline and week 6 was determined in each group. 


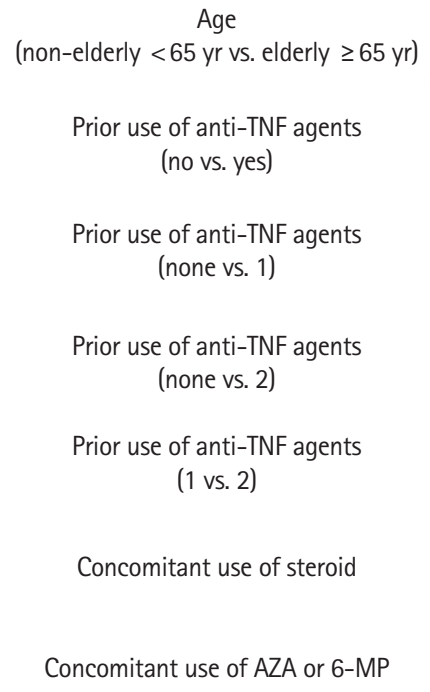

Concomitant use of steroid

Concomitant use of AZA or 6-MP

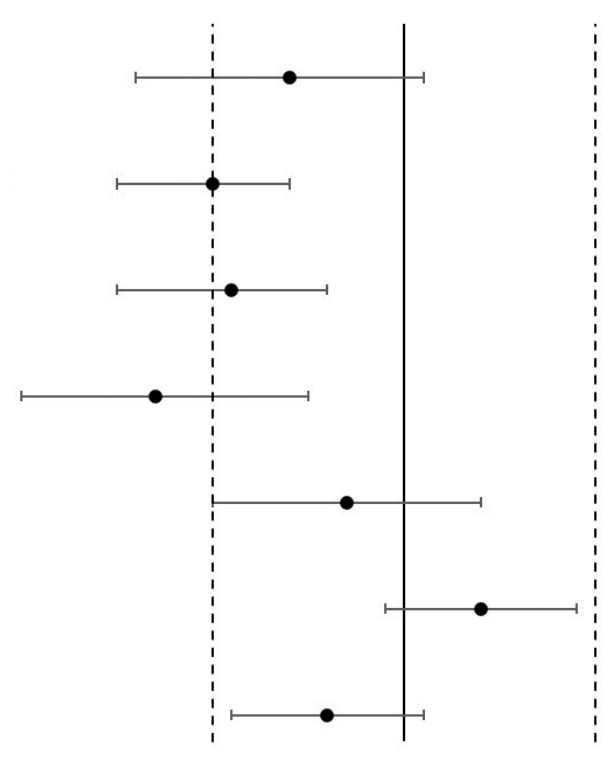

$\begin{array}{lll}-1.0 & 0.0 & 1.0\end{array}$
Means difference $(95 \% \mathrm{Cl})$

$-0.6(-1.4,0.1)$

$-1.0(-1.5,-0.6)$

$-0.9(-1.5,-0.4)$

$-1.3(-2.0,-0.5)$

$-0.3(1.0,0.4)$

0.360

$0.4(-0.1,0.9)$

0.110

$-0.4(-0.9,0.1)$

0.090

Mean difference of change from baseline of pMayo score

Supplementary Fig. 3. Univariable analysis of the factors associated with changes in the partial Mayo (pMayo) score. The patient factors affecting the effectiveness of golimumab (GLM) treatment were determined by forest plots analysis. Change in the pMayo score from baseline to week 6 was used to evaluate GLM effectiveness. Non-elderly or elderly classified as patients aged $<65$ or $\geq 65$ years, respectively. The $P$-value was calculated using the independent $t$-test. ${ }^{a} P<0.05$. TNF, tumor necrosis factor; AZA, azathioprine; 6 -MP, 6 -mercaptopurine; $\mathrm{Cl}$, confidence interval. 


\section{REFERENCE}

1. Matsuoka K, Kobayashi T, Ueno F, et al. Evidence-based clinical practice guidelines for inflammatory bowel disease. J Gastroenterol 2018;53:305-353. 